\title{
A Democratic Head Election Strategy for Clustering in Wireless Sensor Network
}

\author{
Sudakshina Dasgupta \\ Department of Information Technology \\ Government College of Engineering \& Textile \\ Technology, Serampore, India
}

\author{
Paramartha Dutta \\ Department of Computer and System Science \\ Visva Bharati University, Santiniketan, India
}

\begin{abstract}
In recent years a lot of work has been done on Wireless Sensor Network (WSN) as it find its application in many applications including environmental monitoring and military field surveillance. In these applications tiny sensor nodes are deployed across the application regions. Data gathered by the sensor nodes are periodically collected by the base stations. As the nodes are deployed and left unattended for a long time it becomes quite difficult to recharge the node batteries. Several WSN applications require only an aggregate value to be reported to the base station. In this case sensors in different regions of the field can collaborate to aggregate their data and provide more accurate reports about their local regions. This type of data aggregation can reduce the communication overhead in the network and can increase the network life time.

In order to support data aggregation through efficient network organization, nodes can be partitioned into a number of small groups called clusters. Each cluster has a coordinator, referred to as cluster head and a number of member nodes. The member nodes report their data to the respective Cluster Heads (CHs). The $\mathrm{CHs}$ aggregate the data and send them to the central base station through other $\mathrm{CHs}$. This process improves the network lifetime which is an important metric to evaluate the network performance. In this type of system the challenging issue is to selection of $\mathrm{CHs}$ in each cluster along with the cluster head rotation to increase network life time and to reduce the communication overhead among the nodes in a WSN. In our work we have proposed an election algorithm to select the $\mathrm{CHs}$ in a democratic fashion by the nodes in the network. The performance of the proposed algorithm has been successfully demonstrated by the simulation of the proposed system.
\end{abstract}

\section{General Terms}

Cluster-head, LEACH, Mean-Shift.

\section{Keywords}

LEACH, Mean Shift, Cluster-Head, Base-Station, Sensor Node, Network Life Time.

\section{INTRODUCTION}

A sensor node is the core component of a WSN. Sensor nodes can take on multiple roles in a network, such as simple sensing, data storage, routing, data processing, etc. [5]. Sensor nodes are typically less mobile, more limited in capabilities, and more densely deployed than mobile ad-hoc networks (MANETs) [1, 3]. This necessitates devising novel energy efficient solutions to some of the conventional wireless networking problems, such as medium access control, routing, self-organization, bandwidth allocation, and security. Exploiting the tradeoffs among energy, accuracy, and latency, and using hierarchical (tiered) architectures are important techniques for prolonging the network lifetime. Hence it is required to partition the nodes in the network in number of clusters and select appropriate Cluster Head in each cluster to enhance the network life time [8].

The figure 1 shows the typical architecture of a cluster based WSN.

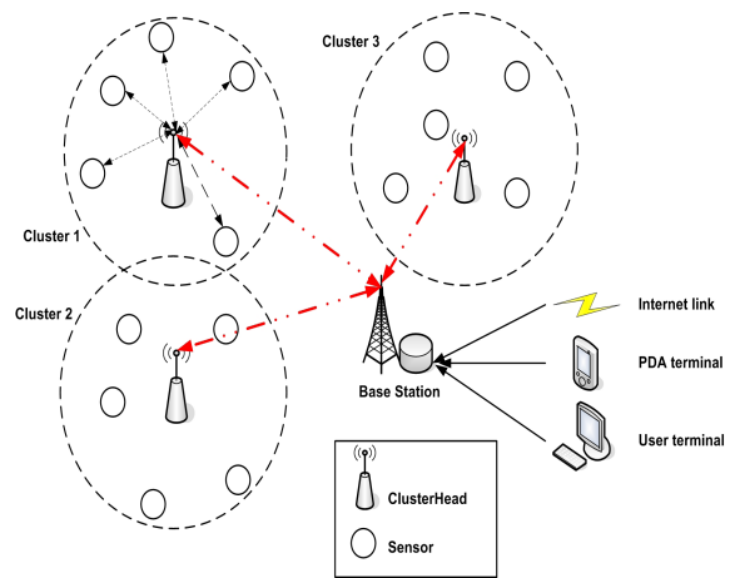

Fig 1.General Sensor Network Architecture

Clusters are the organizational unit for WSNs. The dense nature of these networks requires the need for them to be broken down into clusters to simplify tasks such a communication [4]. These clusters are responsible to communicate data as well as sensing machine failure, biological detection, inventory tracking, environmental monitoring etc by the sensor nodes through the Cluster-Head [7]. At first this cluster-head collects data and then transmits the data to other nodes of the same cluster. This cluster head is responsible for cluster to cluster communication (i.e. Communication between two or more cluster).Cluster Head performs inter and intra cluster communication. The base station is at the upper level of the hierarchical WSN. It provides the communication link between the sensor network and the end-user $[8,9]$.The selection of Cluster Heads in the WSN is a challenging issue as efficient cluster head selection algorithm can improve the life time of the network and can reduce the communication overhead in the network.

In our work we have proposed a democratic cluster head election algorithm to select cluster heads in each round, which can eventually increase the network life time. We have considered the following node parameters in the election process:

i) Distance: It is measured as the Euclidian distance between two nodes. It is desirable that the node with smaller distance from other nodes in the cluster will get maximum vote. 
ii) Remaining Energy: To send and receive data by a node some amount of energy is depreciated. Hence the proposed algorithm has taken care of remaining energy of a node to elect it as the cluster head.

\section{ARCHITECTURE OF WIRELESS SENSOR NETWORKS}

The architecture of a Wireless Sensor Network can be classified in the following groups as shown in figure 2 .

- Single Hop without clustering: In this architecture, without forming any sort of clusters, each sensor node deployed in the network can directly communicate with the sink/base station.

- Multi Hop without Clustering: In this architecture, nodes send their data destined ultimately for sink/base station through their neighbour nodes. Clustering is not required here for network operation.

- Single Hop with Clustering: In this architecture, nodes are first grouped together to from clusters then send their data to their respective cluster-head which in turn forwards the aggregated data to sink/base station.

- Multi Hop with Clustering: In this architecture, cluster heads send their aggregated data destined ultimately for sink/base station through neighbouring cluster-heads [13].

The proposed scheme belongs to the "single hop with clustering" architecture $[7,8]$ to transmit its data to sink/base station.

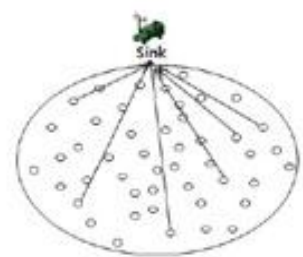

(a) Single-hop flat model

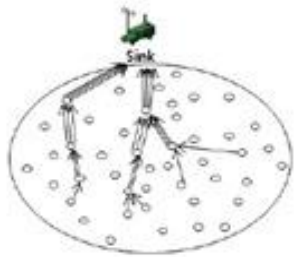

(c) Multi-hop flat model

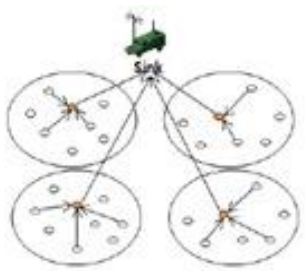

(b) Single-hop clustering model

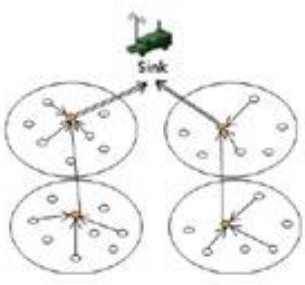

(d) Multi-hop clustering model
Fig 2. Classification of WSN Topologies

\section{RELATED WORKS}

In a hierarchical network, such as a cluster-based network, sensors organize themselves into clusters [11, 12, 17 ]. Each cluster in the network has a cluster head. The cluster heads may or may not be powerful than the other sensors. Each cluster head manages the sensor nodes in its cluster for communication between the cluster and the base station. Communication between cluster head and base station may be multiple hops through other cluster heads. In the next section we describe some cluster based scheduling algorithms.

\subsection{LEACH}

Low-Energy Adaptive Clustering Hierarchy (or LEACH) was one of the first major improvements on conventional clustering approaches in wireless sensor networks. LEACH provides a balancing of energy usage $[2,14]$ by random rotation of cluster heads. The algorithm is also organized in such a manner that data-fusion can be used to reduce the amount of data transmission. The decision of whether a node elevates to cluster head is made dynamically at each interval. The elevation decision is made solely by each node independent of other nodes to minimize overhead in cluster head establishment. This decision is a function of the percentage of optimal cluster heads in a network (determined a priori on application), in combination with how often and the last time a given node has been a cluster head in the past. The threshold function is defined as:

otherwise

$$
\mathrm{T}(\mathrm{n})=\mathrm{P} /[1-\mathrm{P}(\mathrm{r} \bmod 1 / \mathrm{P})] \text { if } \mathrm{n}(-\mathrm{G}
$$

$$
\mathrm{T}(\mathrm{n})=0
$$

Where $\mathrm{n}$ is the given node, $\mathrm{P}$ is the a priori probability of a node being elected as a cluster head, $\mathrm{r}$ is the current round number and $\mathrm{G}$ is the set of nodes that have not been elected as cluster heads in the last 1/P rounds. Each node during cluster head selection will generate a random number between 0 and 1. If the number is less than the threshold $(\mathrm{T}(\mathrm{n}))$, the node will become a cluster head. Following elevation to cluster head, the new cluster head will broadcast its status to neighbouring nodes. These nodes will then determine the optimal cluster head (in terms of minimum energy required for transmission) and relay their desire to be in that particular cluster. The broadcast messages as well as cluster establishment messages are transmitted using CSMA (Carrier Sense Multiple Access) to minimize collisions. Following cluster establishment, cluster heads will create a transmission schedule and broadcast the schedule to all nodes in their respective cluster. The schedule consists of TDMA slots for each neighbouring node. This scheduling scheme allows for energy minimization as nodes can turn off their radio during all but their scheduled time-slot. The operation of LEACH algorithm is shown in Figure 3.

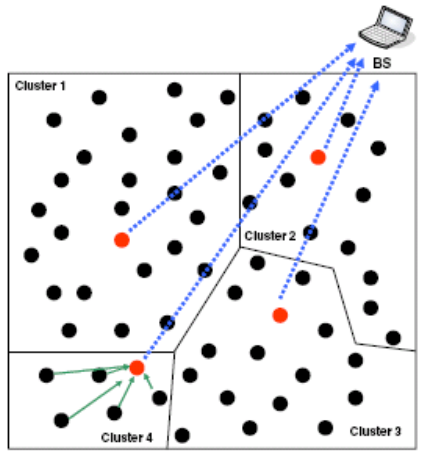

Fig 3. LEACH operation

The features of LEACH algorithm is summarized as follows:

1. Self organizing, adaptive clustering protocol.

2. Even distribution of energy among the sensor nodes.

3. Application specific data processing

4. Cluster head communicates data to the base station.

5 . Dynamic cluster formation.

6 . Randomized rotation of cluster head after each round. 


\subsection{TL-LEACH}

Two-Level Hierarchy LEACH (or TL-LEACH)[19] is a proposed extension to the LEACH algorithm. It utilizes two levels of cluster heads (primary and secondary) in addition to the other simple sensing nodes. In this algorithm, the primary cluster head in each cluster communicates with the secondaries, and the corresponding secondaries communicate with the nodes in their sub-cluster [15]. Data-fusion can also be performed as in LEACH. In addition, communication within a cluster is still scheduled using TDMA time-slots. The organization of a round will consist of first selecting the primary and secondary cluster heads using the same mechanism as LEACH, with a priori probability of being elevated to a primary cluster head less than that of a secondary node. Communication of data from source node to sink is achieved in two steps.

1) Secondary nodes collect data from nodes in their respective clusters. Data-fusion can be performed at this level.

2) Primary nodes collect data from their respective secondary clusters. Data-fusion can also be implemented at the primary cluster head level. The two-level structure of TL-LEACH reduces the amount of nodes that need to transmit to the base station, effectively reducing the total energy usage.

\subsection{Mean Shift Algorithm}

Mean Shift is a powerful and versatile non parametric iterative algorithm that can be used for lot of purposes like finding modes, clustering etc. Mean Shift was introduced by Fukunaga and Hostetler [16] and has been extended to be applicable in field like computer vision. Mean Shift considers feature space as an empirical probability density function. If dense regions or clusters are present in the feature space, then they correspond to the mode (or local maxima) of the probability density function.

For each data point, Mean Shift associates it with the nearby peak of the data set's probability density function. For each data point, Mean Shift defines a window around it and computes the mean of the data point. Then it shifts the centre of the window to the mean and repeats the algorithm till it converges. After each iteration we can consider that the window shifts to a more denser region of the data set [10]. At the high level Mean Shift can be specified as follows:

1.Fix a window around each data Point.

2.Compute the mean of data within the window.

3.Shift the window to the mean and repeat till the convergence.

\section{PROPOSED WORK}

The existing Cluster Head selection algorithms like LEACH does not consider the remaining energy of the sensor nodes for selection of the cluster head. Secondly they require the cluster heads has to be rotated in each round. This can lead to the wastage of the battery power of the nodes and can reduce the life time of the network.

In our work we have considered the single-hop cluster-based WSN with hundreds or thousands sensor nodes dispersed in a field as shown in Fig.4. Base Station(BS), an observer, is located outside the field remotely. The observed field is composed of several clusters. Each cluster has one $\mathrm{CH}$ which acts as a local control centre to coordinate the data transmissions. Here we have proposed a democratic election procedure to find the cluster heads. The followings are the different election parameters to elect the cluster head for each cluster. In the proposed democratic election procedure each node will have the right to vote to select a leader within its cluster using a voting procedure and after that the elected node becomes the cluster head of that cluster. The major parameters[18] to elect cluster heads with in the clusters are described below.

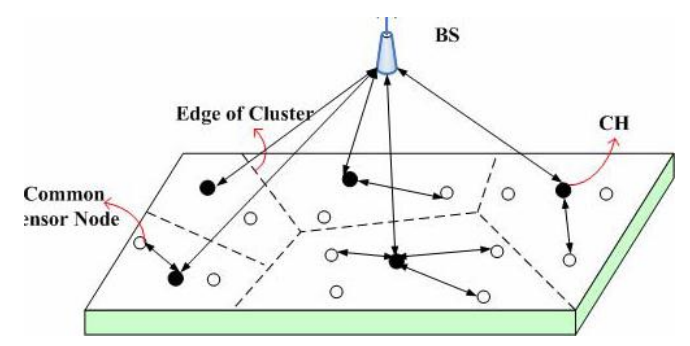

Fig 4. Cluster-based WSN architecture

1. Distance factor: It is the distance between a node to all other nodes in that cluster.

2. Internal energy: It is the remaining energy of a node after a number of packet transmission and reception by that node.

\section{Proposed Algorithm:}

Step 1: Initialization Deploy the sensor nodes randomly over a deployment area. Step 2: Cluster Formation Initially form the clusters using Fuzzy C-Means algorithm. Step 3: Cluster Head Selection

For each cluster $\mathrm{C}_{\mathrm{i}}$ in the network

For each node $i$ in the cluster $C_{i}$

i) Calculate the Euclidean distance $\left(\mathrm{d}_{\mathrm{ij}}\right)$ between node $\mathrm{i}$ and all other nodes in a cluster

ii) Calculate the remaining energy $e_{i}$ of the node $i$

iii) Calculate the voting amount $V_{i}$ for node $i$ using following formula:

$$
V i=\sum_{\substack{i=1 \\ i \neq j}}^{\mathrm{n}} \mathrm{d}_{\mathrm{ij}}+\mathrm{e}_{\mathrm{i}}
$$

End For

iv) The node having the maximum vote i.e. maximum value of $V_{i}$ is elected as the new Cluster Head of cluster $C_{i}$ for that round.

End For

Step 4. Steady State Data Transmission

Nodes in the network continue to send data to the Base Station.

Step 5. Cluster Head Rotation

Go to Step 3 when the remaining energy of a cluster head falls below the threshold value.

Step 6. End

\section{EXPERIMENT AND RESULTS}

In our work we have carried out the simulation within a $200 \times 200 \mathrm{~m}^{2}$ area with 250 sensors deployed randomly within that area assuming that they are deployed in clusters with inter cluster communication will happen only through cluster heads 
of the respective clusters. The base station is positioned at (50, 50). The Snapshot of the system is given in figure 5.

A node is considered to be a "dead" node if its energy level is Zero and can be put is "sleep" mode if less than $10 \%$ of its initial energy is remained.

We have analyzed the performance of Leach algorithm and Mean Shift algorithm with our proposed algorithm in terms of communication overhead, the number of communication rounds (network lifetime), total amount of energy dissipated in the system over time and number of dead nodes in the system over time using simulator based on MATLAB.

For a node in the sensing state, packets are generated at a constant rate of 1 packet/sec. Considering the fact that once the sensors are deployed in the system they are fixed and mobility is disabled, we have calculate the distance (d) between the sensors with in a cluster using the concept of Euclidian distance. Again we also consider that the system is contention and error free and hence the sensors need not retransmit data. In these experiments, each node begins with an initial energy of 0.5 joule and unlimited amount of data can be sent to the Base station via cluster head.

In table 1 we summarize the parameters for experiments:

\section{Table 1: Network parameters used for the experiments}

\begin{tabular}{|l|l|}
\hline Parameter & Value \\
\hline Network Size & $200 \times 200 \mathrm{~m}^{2}$ \\
\hline Number of Sensors & 250 \\
\hline Base Station Location & $(50,50)$ \\
\hline Packet Generating Rate & $1 \mathrm{packet} / \mathrm{Sec}$ \\
\hline EElec & $50 \mathrm{~nJ} / \mathrm{bits}$ \\
\hline عfs & $10 \mathrm{pJ} / \mathrm{bits} / \mathrm{m}^{2}$ \\
\hline Initial Energy & $0.5 \mathrm{Jules}$ \\
\hline $\begin{array}{l}\text { Data packet size(nodes to } \\
\text { cluster head with in a } \\
\text { cluster) }\end{array}$ & 550 bytes \\
\hline $\begin{array}{l}\text { Data packet size(cluster } \\
\text { head to base station) }\end{array}$ & $\begin{array}{l}600 \text { bytes } \\
(50 \text { bytes extra for packet } \\
\text { header) }\end{array}$ \\
\hline
\end{tabular}

For the first experiment we have compared the number of dead nodes in the system with time as well as no. of rounds and in the second experiment we have compared the number of alive nodes in the system with time. We have compared the cases for LEACH, Mean Shift and the proposed algorithm. It can be observed that the number of dead nodes is less and the number of alive nodes is more in case of the proposed algorithm as the time of simulation increases in compare with Mean Shift and LEACH algorithm. This is because the LEACH and Mean Shift algorithm does not take care of remaining energy of a node while selecting a cluster head. For the same reason the result is obvious in the second experiment.

Figure 6 and figure 7 depict the result of first and second experiment respectively.

In last experiment we have calculated the frequency of cluster head rotation with time for both algorithms. It is observed that in case of the proposed algorithm the cluster head changes less frequently and on demand leading to more stable and energy efficient system of networks. The result of the last experiment is depicted in figure 8 .

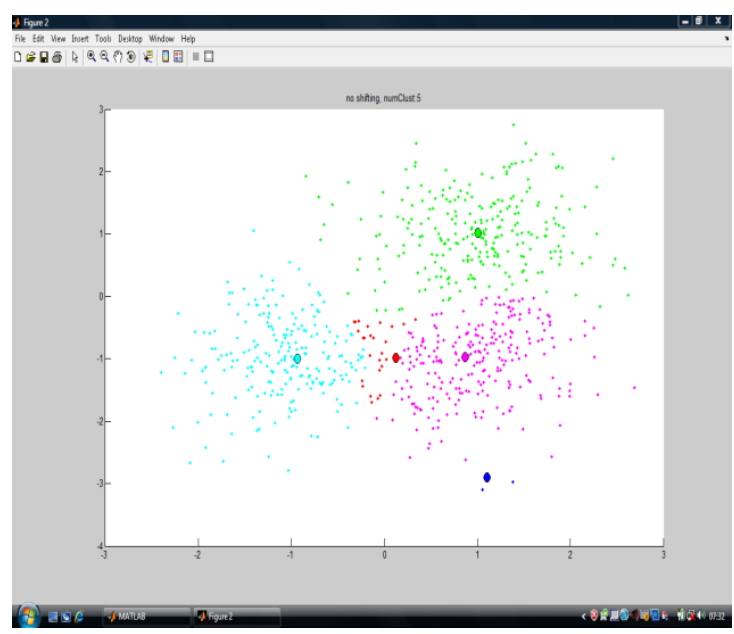

Fig 5. Initial Cluster formation in the WSN

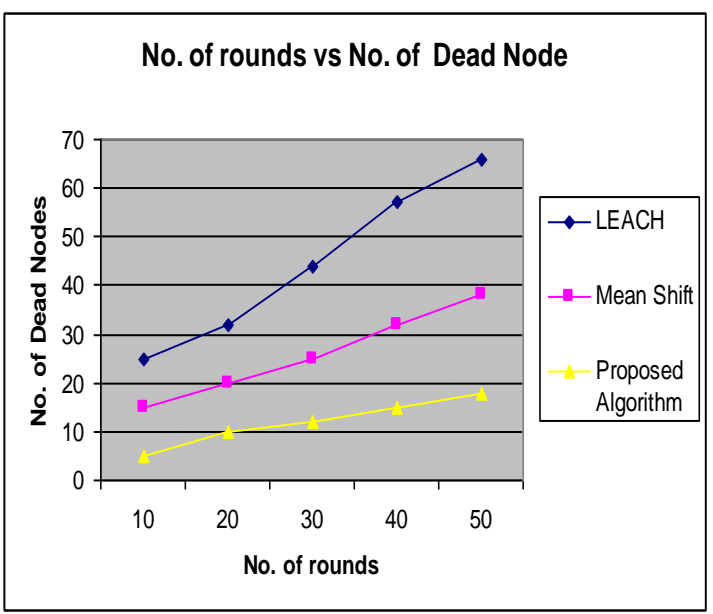

Fig 6. Number of rounds vs. Number of dead nodes

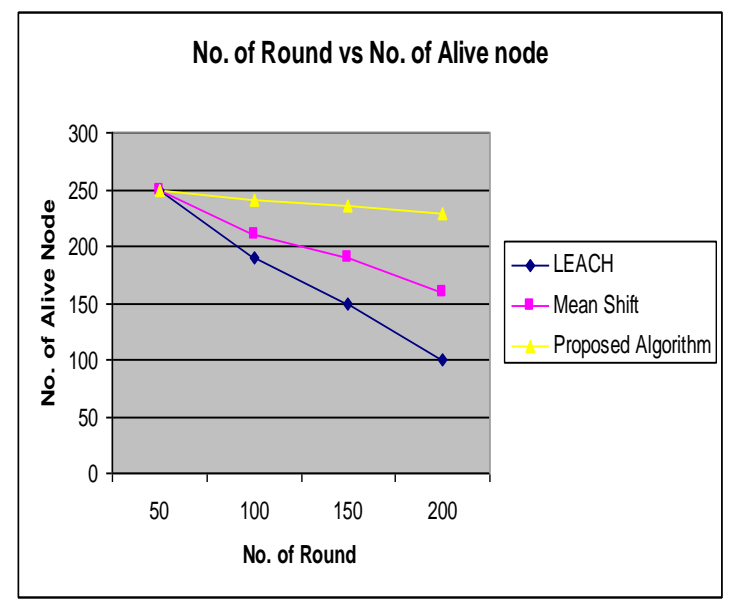

Fig 7. Number of rounds vs. Number of alive nodes 


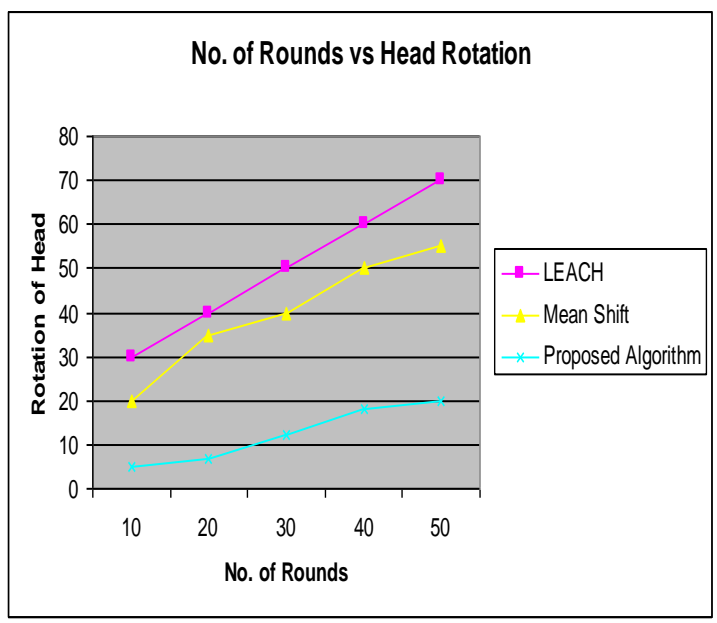

Fig 8. Number of rounds vs. cluster head rotation

\section{CONCLUSION}

Efficient cluster head selection strategy is an important problem in the field of WSN. The proposed scheme is basically a modification of an existing Mean Shift algorithm for cluster head selection. The algorithm performs better than the existing algorithm because it does not necessarily elect the central node as cluster head and the cluster head is only rotated if the remaining energy of that node falls below certain threshold level. The next cluster head is also elected by the other nodes in the cluster in a democratic way, which results in a high network life time.

\section{REFERENCES}

[1] I. F. Akyildiz,W. Su, Y. Sankarasubramaniam, and E. Cayirci Aug 2002. A Survey on Sensor Netowrks. IEEE Communications Magazine, vol. 40, no. 8, pp. 102114.

[2] W. R. Heinzelman, A. Chandrakasan, and H. Balakrishnan Jan 2000. Energy Efficient Communication Protocol for Wireless Micro Sensor Networks. Proceedings of IEEE HICSS. Jan 2000.

[3] C. F. Chiasserini, I. Chlamtac, P. Monti, and A. Nucci Feb 2002. Energy Efficient Design of Wireless Ad Hoc Networks. Proceedings of European Wireless.

[4] S. Bandyopadhyay and E. J. Coyle April 2003.An Energy Efficient Hierarchical Clustering Algorithm for Wireless Sensor Networks. IEEE INFOCOM.

[5] A. Mainwaring et al. 2002. Wireless Sensor Networks for Habitat Monitoring. Proceedings of the 1st ACM International Workshop on WSN.

[6] C. Intanagonwiwat et al. Feb. 2003. Directed Diffusion for Wireless Sensor Networking. IEEE/ACM Transaction on Networking, vol. 11, no. 1.

[7] K.Ramesh and Dr K.Somasundaram November 2011 . A Comparative Study Of Clusterhead Selection Algorithm In Wireless Sensor Networks. International Journal of Computer Science \& Engineering Survey (IJCSES) Vol.2, No.4.
[8] Kiran Maraiya, Kamal Kant, Nitin Gupta June 2011.Efficient Cluster Head Selection Scheme for Data Aggregation in Wireless Sensor Network.International Journal of Computer Applications (0975 - 8887) Volume 23- No.9,

[9] Yaoyao Yin, Juwei Shi, Yinong Li , Ping Zhang. Cluster Head Selection Using Analytical Hierarchy Process For Wireless Sensor Networks.

[10] Quan Zhou, Xiaowei Li, Yongjun Xu Mean Shift Based Collaborative Localization with Dynamically Clustering for Wireless Sensor Networks volume: 2 ,Page(s): 6670 .

[11] S. Bandyopadhyay , E. Coyle April 2003. An energy efficient hierarchical clustering algorithm for wireless sensor networks. Proceedings of the 22nd Annual Joint Conference of the IEEE Computer and Communications Societies, San Francisco, California.

[12] O. Younis, S. Fahmy 2004. HEED: A Hybrid, EnergyEfficient, Distributed clustering approach for Ad Hoc sensor networks. IEEE Transactions on Mobile Computing 3 (4) 366-379.

[13] W. B. Heinzelman, A. P. Chandrakasan, H. Balakrishnan 2002. Application specific protocol architecture for wireless microsensor networks. IEEE Transactions on Wireless Networking.

[14] Wendi Rabiner Heinzelman, Anantha Chandrakasan, and Hari Balakrishnan, $.2000 \quad$ Energy-Efficient Communication Protocol forWireless Microsensor Networks. Proceedings of the 33rd Hawaii International Conference on System Sciences.

[15] Deng Zhixiang, Qi Bensheng 2007 Three-layered routing protocol for WSN based on LEACH algorithm. Wireless Mobile and Sensor Networks,.Page(s):72-75.

[16]Yizong Chen . August 1995 Mean Shift, Mode Seeking, and Clustering. IEEE Transactions on pattern analysis and machine intelligence, Vol. 17, No, 8 .

[17] Vivek Katiyar, Narottam Chand, Surender Soni ,2011. A Survey on Clustering Algorithms for Heterogeneous Wireless Sensor Networks. . Int. J. Advanced Networking and Applications Volume: 02, Issue: 04, Pages: 745-754.

[18] Kiran Maraiya, Kamal Kant, Nitin Gupta,2011. Efficient Cluster Head Selection Scheme for Data Aggregation in Wireless Sensor Network. International Journal of Computer Applications (0975 - 8887) Volume 23- No.9. June 2011

[19] Ankita Joshi , Lakshmi Priya.M. A Survey of Hierarchical Routing Protocols in Wireless Sensor Network , MES Journal of Technology and Management 